\title{
Emergency management of dental trauma: a survey of public knowledge, awareness, and attitudes in Al-Madinah Al-Munawwarah
}

This article was published in the following Dove Press journal:

Clinical, Cosmetic and Investigational Dentistry

\section{Ibrahim Mohammedali \\ Nourwali \\ Abdulrahman Khaleel Maddhar ${ }^{2}$ \\ Bayan Hussain Alsaati ${ }^{3}$ \\ Rahaf Abed Alhazmi ${ }^{2}$ \\ Shahad Mohammed AlAyoubi ${ }^{2}$ \\ Shaima Sabri AlHarbi (D) ${ }^{3}$ \\ 'Oral \& Maxillofacial Surgery, Faculty of Dentistry, Taibah University, Medina, Saudi Arabia; ${ }^{2}$ Faculty of Dentistry, Taibah University, Medina, Saudi Arabia; ${ }^{3}$ Faculty of Dentistry, Taibah University, Medina, Saudi Arabia}

Video abstract

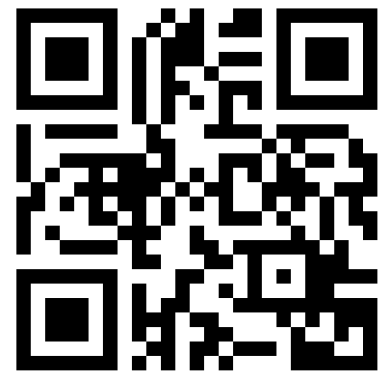

Point your SmartPhone at the code above. If you have QR code reader the video abstract will appear. Or use: https://youtu.be/a]XQe3FFZRA
Correspondence: Shaima Sabri AlHarbi Faculty of Dentistry, Taibah University, 3587 Hassan Ibn Thabit Street-Al Iskan, Medina 42317-7279, Saudi Arabia Tel +966551879359

Email shimasabria@gmail.com
Background: Dental trauma is one of the most commonly encountered dental emergencies. The prognosis is determined by the appropriate intervention and immediate management of the traumatized teeth. Thus, public awareness is a leading determinant of treatment success. The purpose of this study was to assess the knowledge, attitudes, and awareness of AlMadinah Al-Munawwarah residents regarding the management of dental trauma.

Methods: This cross-sectional observational study was conducted between December 2016 and January 2018. A two-part self-administered questionnaire comprising 13 closed-ended questions was used. Part one contained questions to elicit demographic data that included information related to the participants' sources of dental trauma. Part two assessed their knowledge and attitudes regarding the most common types of dental trauma. The data were analyzed with IBM SPSS Statistics for Windows, Version 22.0 software.

Results: A total of 598 participants were included in this study. The results indicated that $64 \%$ of the participants knew where to go to receive treatment for dental trauma, and $74 \%$ were aware of the differences in the management of the primary and permanent teeth after trauma. A majority (83\%) did not have any information about these issues; however, 83\% were interested in learning about the appropriate management of dental trauma.

Conclusion: The study participants were found to have low levels of knowledge. A majority had never received information about the management of dental trauma or avulsed teeth. Education programs are necessary for promoting positive attitudes by increasing the knowledge about dental trauma management.

Keywords: avulsion, dental trauma, awareness, knowledge, management

\section{Introduction}

Dental trauma is highly prevalent. ${ }^{1}$ It can affect children and teenagers at home, at school, or outdoors. Dental trauma most frequently occurred in the home, and enamel trauma was the most common type. The maxillary central incisors were the most commonly affected, followed by the maxillary lateral incisors and canines. ${ }^{2}$ These injuries are often seen in conjunction with facial trauma, which poses esthetic concerns to patients. ${ }^{3}$ The severity of traumatic dental injuries can range from minor cases of enamel chip to extensive injuries involving the supporting structures or dental avulsion. ${ }^{4}$ Inflammatory root resorption and eventual exodontia could result from the degeneration of the periodontal ligament cells and pulp necrosis if the above-mentioned factors are unfavorable. ${ }^{5}$ Not only does anterior tooth trauma have a negative esthetic effect, but it also threatens the capabilities for pronunciation 
and mastication. Furthermore, it is evident that dental trauma can negatively affect psychological and mental wellbeing. Sudden life transitions can profoundly influence social, family, and psychological life. ${ }^{6}$ In sum, dental injuries are considered to be the most serious dental condition experienced by children. ${ }^{7}$

In cases of dental trauma, the prognosis is determined by the appropriate intervention and immediate emergency management of the traumatized teeth. ${ }^{8}$ For example, in cases of avulsed teeth, the prognosis depends mainly upon the immediate management at the time of the emergency. Other factors include extra oral dry time, the reimplementation of the tooth before the patient's arrival at the clinic, and the storage of the tooth in a physiologic storage medium or osmolality-balanced medium. ${ }^{9}$ Therefore, public awareness of the immediate management of dental trauma plays a major role in the treatment and prognosis. Education programs must be implemented to increase awareness to enhance the prognosis for dental trauma.

Few studies have evaluated public awareness of this issue in Al-Madinah Al-Munawwarah city, Saudi Arabia. Therefore, the present study aimed to assess the level of knowledge and the attitudes of Al-Madinah Al-Munawwarah residents regarding dental trauma management.

\section{Methods}

This cross-sectional observational study was conducted between December 2016 and January 2018. The data were collected through a validated Arabic anonymous self-report questionnaire, ${ }^{10,11}$ which was distributed to residents who visited the community-based awareness camps run by Taibah Medical Clube in 2017. Located in the Western region of the Kingdom of Saudi Arabia, Al-Madinah Al-Munawwarah has 13 administrative regions. The population is $1,183,205$ : $655,470(53.7 \%)$ male and 527,735 (46.3\%) female. ${ }^{12}$

The two-part questionnaire comprised 13 questions. The first section investigated the participants' demographics and the sources of their dental trauma. The second section, which contained closed-ended questions, assessed their knowledge and attitudes regarding common dental trauma. Participation was voluntary, and the subjects were diverse in terms of age, gender, and education to ensure that the sample would be as representative as possible.

A convenience sample of 600 Al Madinah AlMunawwarah residents aged 18 and older were recruited. According to the sample size calculator, a sample size of 600 was needed to achieve a 95\% confidence level. Approximately 700 questionnaires were distributed to account for the exclusion criteria and the possibility of missing data.
After the completed questionnaires were received, the data were entered and coded in IBM SPSS Statistics, Version 22.0 software. A descriptive analysis was performed, and the mean and standard deviation were determined. The project did not involve the collection of sensitive information, and there were no intellectual property issues. Thus, no specific management plan was developed. The Taibah University College of Dentistry Research Ethics Committee approved this study, with a waiver of informed consent from the participants because no sensitive information was collected and the study posed no risk.

\section{Results}

A total of 652 completed questionnaires were collected (response rate $=93 \%$ ). After the exclusion of the questionnaires with missing data and those of participants younger than 18 years old, 598 questionnaires remained for analysis. The participants' demographic data are presented in Table 1. A majority (78\%) were female, 89\% were 19-29 years old, and $82 \%$ had a college education.

Most of the participants $83 \%(n=496)$ indicated that they had not received any information and had no knowledge about the management of dentoalveolar trauma. The remainder ( $17 \% ; n=102$ participants) had received information from social media, 52\% $(n=63 / 102)$, followed by the internet $(37 \% ; n=44)$, the journals and magazines were the least cited source $(5 \% ; n=6)$. Most of the participants $(83 \%)$ were interested in learning about the appropriate management of dentoalveolar trauma, and they preferred to receive this information through television $(30 \%)$ or social media (28\%; Table 2).

Table I Demographic characteristics of respondents $(\mathrm{N}=598)$

\begin{tabular}{|l|l|}
\hline Demographics/characteristics & No. (\%) of respondents \\
\hline Gender & \\
Fale & $142(22 \%)$ \\
\hline Age & $501(78 \%)$ \\
\hline $19-29$ & \\
$30-39$ & $530(89 \%)$ \\
$40-49$ & $46(8 \%)$ \\
$>50$ & $20(3 \%)$ \\
\hline Education level & $2(0.3 \%)$ \\
High school & \\
College & $95(16 \%)$ \\
Post-grade & $489(82 \%)$ \\
\hline
\end{tabular}


Table 2 Assessment of participants' behaviors regarding education on managing dentoalveolar trauma

\begin{tabular}{|l|l|}
\hline $\begin{array}{l}\text { Are you interested in receiving information about dealing } \\
\text { with dentoalveolar trauma? (If you answer "Yes," answer the } \\
\text { next question) }\end{array}$ \\
\hline Yes & $498(83 \%)$ \\
No & $100(17 \%)$ \\
\hline How would you prefer to get this information? \\
\hline Books & $90(14 \%)$ \\
Television show & $180(30 \%)$ \\
Social media & $165(28 \%)$ \\
Other & $63(11 \%)$ \\
\hline
\end{tabular}

In the second section, which assessed the participants' knowledge and awareness of the appropriate management of dentoalveolar trauma, approximately $64 \%$ reported that the suitable place to deal with such issues was the dental clinic. Approximately 33\% preferred the emergency room, and $65 \%$ indicated that this should occur immediately. There was some variation in the opinions about the management of an avulsed tooth: $36 \%$ did not know what to do with a broken or avulsed tooth, and approximately $22 \%$ stated that they would place the tooth in a liquid medium, such as saliva, milk, or water, and take it to the dental clinic. Approximately 56\% of the participants stated that they could replant the avulsed tooth in its location inside the patient's mouth.

Regarding the proper management of patients with cut wounds and facial bleeding, $43 \%$ of the participants reported that they would stanch the bleeding by first applying pressure with gauze. Approximately $28 \%$ did not know how to properly manage this situation. Finally, most of the participants (74\%) reported that there were differences in the management of primary and permanent teeth after dentoalveolar trauma (Figure 1).

\section{Discussion}

The goal of this study was to explore the levels of knowledge and the attitudes of a group of Al-Madinah Al-Munawwarah residents regarding the management of dentoalveolar trauma. The findings were expected. This suggests that the participants had relatively less knowledge about the management of traumatic dental injuries. The findings confirm those of several previous studies of mothers in Mosul, Iraq, ${ }^{13}$ teachers in the city of Mathura, ${ }^{14}$ health teachers in Jordan's schools, ${ }^{15}$ and physical education teachers in Bangalore's urban schools. ${ }^{16}$ However, a majority of the subjects in the present study were aware of the initial management of patients with cut wounds and facial bleeding: the application of pressure with gauze to stanch the bleeding.

Kaul et al conducted a study of school teachers in Kolkata. In contrast to some previous studies about the management of dental injuries, this study found that the participants had a good level of knowledge. ${ }^{10}$

Assessing public awareness of the appropriate place and time for seeking help after dentoalveolar trauma is of the utmost importance. In the present study, a majority of the participants who sustained dental trauma, or whose children did, indicated that they would go to a dental clinic. Most recognized the urgency of seeking immediate professional assistance. These findings are representative of the appropriate public attitudes. They also highlight the role of general dentists, who are often the first to see these patients. They should therefore be equipped with the knowledge and means to manage them appropriately. ${ }^{17}$

Avulsion is one of the most severe types of dental injuries. The clinical prognosis is contingent upon the application of the appropriate first aid measures and the promptness with which dental care is received. ${ }^{9,18}$ Thus, particular attention was paid to the management of avulsed teeth. The present assessment indicated that there were variations in the participants' approaches to dealing with avulsed teeth. Specifically, a majority did not know what should be done. These findings are similar to those of Hashim, who reported a low level of knowledge of dental trauma management, especially avulsed teeth, among mothers in Ajman. ${ }^{19}$

One of the most alarming revelations was the participants' responses to specific questions about their knowledge of the possibility for the replantation of an avulsed tooth. Half of them did not realize that this was a possibility. Consequently, these findings can inform the design of educational materials for community-based awareness programs. A majority of the subjects in this study recognized that cut wounds and facial bleeding could be initially managed by the application of pressure with gauze to stanch the bleeding.

These findings regarding the low awareness of the management of avulsed teeth and the possibility of reimplantation is comparable to those of other studies For example, in their cross-sectional survey of school teachers in the city of Davangere, Prasanna et al found that there was inadequate knowledge about the management of avulsed teeth. A majority of the participants did not feel capable of replanting an avulsed tooth. ${ }^{20}$ Of course, the 
What is the proper place to deal with dentoalveolar trauma?

What is the proper time to treat dentoalveolar trauma?

How to deal with broken/ Avulsed tooth after dentoalveolar trauma?

Can you replant avulsed tooth inside the patient mouth?

What is the proper management to deal with patient with cut wound and bleeding of face?

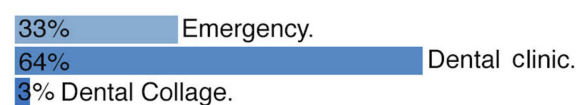

$65 \%$ Immediately.
$7 \%$ After bleeding.
$8 \%$ If the patient feel pain.
$1 \%$ Anytime according to patient desire.
$6 \%$ Upon 24 hours.
$13 \%$ I don't know.

$19 \%$ The broken/ Avulsed tooth is useless, ignore it.

$21 \%$ I will keep the broken/ Avulsed tooth gauze and bring it to clinic.

$22 \% \quad$ will keep the broken/ Avulsed tooth in water, saliva or milk and bring it to clinic.

$2 \%$ l will wash the broken/ Avulsed tooth and trying to replant it.

36\% I don't know.

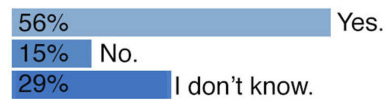

$43 \% \quad$ Stop the bleeding by applying a gauze.

$3 \%$ Stop the bleeding by swapping tissue. $18 \%$ Go to the clinic immediately.

$8 \%$ Go to the hospital for anti-tetanus dose.

28\% I don't know.

Is there any different between management of primary and permeant teeth?

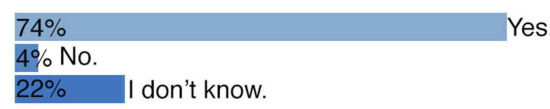

Figure I Assessment of participants' knowledge and awareness about dental trauma management.

variations in the inclusion criteria for the study participants could have contributed to the rate differences between the present study and that of Prasanna et al The present study included subjects with different education levels and occupations, and the Prasanna et al study participants were all primary school teachers.

The ability to distinguish between deciduous and permanent teeth at the scene of a dental injury is crucial. In the present study, most of the respondents (74\%) were aware that there were differences in managing primary and permanent teeth after dental trauma. This contrasts with the findings of Young et al Most of the subjects (70\%) in that study lacked the confidence to distinguish between the two types of teeth. ${ }^{4}$

Despite the frequent occurrence of dental trauma and the access to information, a majority of the participants had never received information about the management of such incidents. It is possible that, generally, less attention is being given to dental first aid protocols. This observation should be considered in the design and implementation of appropriate and engaging awareness programs. It must be noted that the participants with knowledge about the management of dental injures had not received their information from social media. This highlights the importance of the selection of effective and widely used platforms for distributing information.

The data obtained from this study can support the design of effective public awareness programs. The findings reveal that the knowledge gap is related to the understanding of the importance of receiving the appropriate information and training regarding basic dental first aid and the options for managing dental trauma. The findings can contribute to the 
design of appropriate and effective community-oriented education and training programs. Al-Madinah Al-Munawwarah residents might have inadequate knowledge about dental trauma management; therefore, more practical training is required for preventing panic and maintaining control in emergencies.

Some limitations must be noted. First is the nonprobability sample. The participant selection procedures in the present study were more effective than those for probability sampling. Nonprobability sampling was considered particularly useful in this exploratory study because the goal was to determine the existence of the problem through the quickest and least expensive methods. It may also be considered an ethical approach to determining whether a problem or issue is worth deeper investigation because fewer participants are needed.

Second, because the methodology was based on convenience sampling, the study is therefore subject to potential bias. Thus, generalization is not possible because the sample is unlikely to be representative of the population under study.

\section{Conclusion}

This study of a group of Al-Madinah Al-Munawwarah residents highlights the lack of knowledge about dental trauma management. The participants did not know how to deal with avulsed teeth, and they had not received information about managing dental trauma. Thus, strategies for improving public knowledge about dental trauma must be developed. The dental authorities should create awareness by including this topic in school curricula. Camps can also be organized, and the public can be educated through engaging platforms that allow for the incorporation of audiovisual aids.

\section{Recommendations}

On the basis of the findings, the following are recommended:

- An increase in the number of community-based awareness programs, particularly those providing information about dental trauma.

- The design of engaging and captivating events to increase awareness and to ensure city-wide information distribution.

- The exploration of effective approaches for improving the delivery of dental first aid training through courses and workshops.

- The use of economical intervention methods, such as posters and social media, to increase awareness about dental trauma.

\section{Acknowledgment}

The authors acknowledge the Taibah Medical Club for data collection.

\section{Disclosure}

The authors report no conflicts of interest in this work.

\section{References}

1. Azami-Aghash S, Azar F, Azar F, et al. Prevalence, etiology, and types of dental trauma in children and adolescents: systematic review and meta-analysis. Iran Univ Med Sci. 2015;29(40):234.

2. Enabulele J, Oginni A, Sede M, Oginni F. Pattern of traumatised anterior teeth among adult Nigerians and complications from late presentation. BMC Res Notes. 2016;9(1). doi:10.1186/s13104-0161871-3

3. Pani S, Eskandrani R, Al-Kadhi K, Al-Hazmi A. Knowledge and attitude toward dental trauma first aid among a sample of emergency room personnel across Saudi Arabia. Saudi J Oral Sci. 2015;2(1):30. doi:10.4103/1658-6816.150591

4. Young C, Wong K, Cheung L. A survey on Hong Kong secondary school students' knowledge of emergency management of dental trauma. PLoS One. 2014;9(1):e84406. doi:10.1371/journal.pone.0084406

5. Soares A, Gomes B, Zaia A, Ferraz C, Souza-Filho F. Relationship between clinical-radiographic evaluation and outcome of teeth replantation. Dent Traumatol. 2008;24(2):183-188. doi:10.1111/ j.1600-9657.2007.00528.x

6. Pennebaker JW. The effects of traumatic disclosure on physical and mental health: the values of writing and talking about upsetting events. In: Posttraumatic Stress Intervention: Challenges, Issues, and Perspectives. 2000:97-114.

7. Lee JY, Divaris K. Hidden consequences of dental trauma: the social and psychological effects. Pediatr Dent. 2009;31(2):96-101.

8. Jindal A, Bhargava S, Bakshi L, Verma R, Beniwal D, Namdev R. Awareness of emergency management of dental trauma. Contemp Clin Dent. 2014;5(4):507. doi:10.4103/0976-237x.142820

9. Andersson L, Andreasen J, Day P, et al. International Association of Dental Traumatology guidelines for the management of traumatic dental injuries: 2. Avulsion of permanent teeth. Dent Traumatol. 2012;28(2):88-96. doi:10.1111/j.1600-9657.2012.01125.x.

10. Kaul R, Jain P, Saha N, et al. Evaluation of knowledge, awareness, and attitude toward emergency dental trauma management among the school teachers of Kolkata. Indian J Dent Res. 2017;28(6):595. doi:10.4103/ijdr.ijdr_118_17.

11. Halawany H, AlJazairy Y, Alhussainan N, AlMaflehi N, Jacob V, Abraham N. Knowledge about tooth avulsion and its management among dental assistants in Riyadh, Saudi Arabia. BMC Oral Health. 2014;14(1). doi:10.1186/1472-6831-14-46.

12. General Authority for Statistics. General Authority for Statistics. Available from: https://www.stats.gov.sa/en. Accessed October 27, 2018.

13. Yassen G, Chin J, Younus M, Eckert G. Knowledge and attitude of dental trauma among mothers in Iraq. Eur Archives Paediatr Dent. 2013;14(4):259-265. doi:10.1007/s40368-013-0059-y

14. Singh M, Yadav P, Ingle N, Kaur N. Evaluation of knowledge and attitude of school teachers about emergency management of traumatic dental injury. J Int Soc Prev Community Dent. 2015;5(2):108 doi:10.4103/2231-0762.155735

15. Al-Jundi S, Al-Waeili H, Khairalah K. Knowledge and attitude of Jordanian school health teachers with regards to emergency management of dental trauma. Dent Traumatol. 2005;21(4):183-187. doi:10.1111/j.1600-9657.2005.00307.x 
16. Chandan G, Mohandas U. Knowledge, attitude and practice in emergency management of dental injury among physical education teachers: A survey in Bangalore urban schools. J Indian Soc Pedodon Prev Dent. 2009;27(4):242. doi:10.4103/0970-4388.57660

17. Beech N, Tan-Gore E, Bohreh K, Nikolarakos D. Management of dental trauma by general practitioners. Aust Fam Physician. 2015;44(12):915.

18. Santos M, Habecost A, Gomes F, Weber J, de Oliveira M. Parent and caretaker knowledge about avulsion of permanent teeth. Dent Traumatol. 2009;25(2):203-208. doi:10.1111/j.1600-9657.2008.00620.x.
19. Hashim R. Investigation of mothers' knowledge of dental trauma management in United Arab Emirates. Eur Arch Paediatr Dent. 2012;13(2):83-86. doi:10.1007/bf03262849.

20. Prasanna S, Giriraju A, Narayan N. Knowledge and attitude of primary school teachers toward tooth avulsion and dental first aid in Davangere City: a cross-sectional survey. Int J Clin Pediatr Dent. 2011;4:203-206. doi:10.5005/jp-journals-10005-1110.

\section{Publish your work in this journal}

Clinical, Cosmetic and Investigational Dentistry is an international, peer-reviewed, open access, online journal focusing on the latest clinical and experimental research in dentistry with specific emphasis on cosmetic interventions. Innovative developments in dental materials, techniques and devices that improve outcomes and patient satisfaction and preference will be highlighted. The manuscript management system is completely online and includes a very quick and fair peer-review system, which is all easy to use. Visit http://www.dovepress.com/testimonials.php to read real quotes from published authors. 